\title{
Enumerative Encoding in the Grassmannian Space
}

\author{
Natalia Silberstein \\ Department of Computer Science \\ Technion-Israel Institute of Technology \\ Haifa 32000, Israel \\ Email: natalys@cs.technion.ac.il
}

\author{
Tuvi Etzion \\ Department of Computer Science \\ Technion-Israel Institute of Technology \\ Haifa 32000, Israel \\ Email: etzion@cs.technion.ac.il
}

\begin{abstract}
Codes in the Grassmannian space have found recently application in network coding. Representation of $k$ dimensional subspaces of $\mathbb{F}_{q}^{n}$ has generally an essential role in solving coding problems in the Grassmannian, and in particular in encoding subspaces of the Grassmannian. Different representations of subspaces in the Grassmannian are presented. We use two of these representations for enumerative encoding of the Grassmannian. One enumerative encoding is based on Ferrers diagrams representation of subspaces; and another is based on identifying vector and reduced row echelon form representation of subspaces. A third method which combine the previous two is more efficient than the other two enumerative encodings.
\end{abstract}

\section{INTRODUCTION}

Let $\mathbb{F}_{q}$ be a finite field of size $q$. The Grassmannian space (Grassmannian, in short), denoted by $\mathcal{G}_{q}(n, k)$, is the set of all $k$-dimensional subspaces of the vector space $\mathbb{F}_{q}^{n}$, for any given two nonnegative integers $k$ and $n, k \leq n$. A code $\mathbb{C}$ in the Grassmannian is a subset of $\mathcal{G}_{q}(n, k)$.

Koetter and Kschischang [1] showed the application of error-correcting codes in $\mathcal{G}_{q}(n, k)$ to random network coding. This application has motivated extensive work in the area [2], [3], [4], [5], [6], [7], [8]. On the other hand, the Grassmannian and codes in the Grassmannian are interesting for themselves [9], [10], [11], [12], [13]. A natural question is how to encode/decode the subspaces in the Grassmannian in an efficient way. To answer this question we need first to give a representation of subspaces, order all of them, and encode/decode them based on this representation and order.

Cover [14] presented a general method of enumerative encoding for a subset $S$ of binary words. Given a lexicographic ordering of $S$, he presented an efficient algorithm for calculating the index of any given element of $S$ (encoding). He also presented an inverse algorithm to find the element from $S$ given its index (decoding). Our goal in this paper is to apply this scheme to all subspaces in a Grassmannian, based on different lexicographic orders.

First, we present the encoding scheme of Cover [14]. Let $\{0,1\}^{n}$ denote the set of all binary vectors of length $n$. Let $S$ be a subset of $\{0,1\}^{n}$. Denote by $n_{S}\left(x_{1}, x_{2}, \ldots, x_{k}\right)$ the number of elements of $S$ for which the first $k$ coordinates are given by $\left(x_{1}, x_{2}, \ldots, x_{k}\right)$.

The lexicographic order is defined as follows. We say that for $x, y \in\{0,1\}^{n}, x<y$, if $x_{k}<y_{k}$ for the least index $k$ such that $x_{k} \neq y_{k}$. For example, $00101<00110$.
Theorem 1: [14] The lexicographic index of $x \in S$ is

$$
\operatorname{ind}_{S}(x)=\sum_{j=1}^{n} x_{j} \cdot n_{S}\left(x_{1}, x_{2}, \ldots, x_{j-1}, 0\right) .
$$

Remark 1: The encoding algorithm of Cover is efficient if $n_{S}\left(x_{1}, x_{2}, \ldots, x_{j-1}, 0\right)$ can be calculated efficiently.

Let $S$ be a given subset and $i$ be a given index. The following algorithm finds $x$ such that $\operatorname{ind}_{S}(x)=i$.

Inverse algorithm [14]: For $k=1, \ldots, n$, if $i \geq$ $n_{S}\left(x_{1}, x_{2}, \ldots, x_{k-1}, 0\right)$ then set $x_{k}=1$ and $i=i-$ $n_{S}\left(x_{1}, x_{2}, \ldots, x_{k-1}, 0\right)$; otherwise set $x_{k}=0$.

Cover [14] also presented the extension of these results to arbitrary finite alphabet. For our purpose this extension is more relevant as we will see in the sequel. The formula for calculating the lexicographic index of $x \in S \subseteq$ $\{1,2,3, \ldots, M\}^{n}$ is as follows.

$$
\operatorname{ind}_{S}(x)=\sum_{j=1}^{n} \sum_{m<x_{j}} n_{S}\left(x_{1}, x_{2}, \ldots, x_{j-1}, m\right) .
$$

Cover didn't prove the correctness of this formula and didn't present the inverse algorithm. We will present some of these omissions for our decodings in the sequel.

In our work we present three different ways for enumerative encoding of the Grassmannian. One is based on Ferrers diagrams ordering; another is based on the identifying vectors combined with the reduced row echelon form ordering; and the third one is a combination of the first two.

The rest of this paper is organized as follows. In Section we discuss different representations of subspaces in the Grassmannian. We define the reduced row echelon form of a $k$-dimensional subspace and its Ferrers diagram. These two structures combined with the identifying vector of a subspace will be our main tools for representation of subspaces. In Section III we define an order of the Grassmannian based on Ferrers diagrams representation and present the first enumerative encoding method. In Section IV we define another lexicographic order of the Grassmannian based on representation of a subspace by its identifying vector and its reduced row echelon form and describe the second enumerative encoding method. In Section $\nabla$ we show how we can combine two encoding methods mentioned above. Finally, in Section VI we summarize our results and discuss further applications of the different orders of the Grassmannian. This leads for further results and problems for future research. 


\section{Representation of Subspaces}

In this section we give the definitions for two structures which are useful in describing a subspace in $\mathcal{G}_{q}(n, k)$, i.e., the reduced row echelon form and the Ferrers diagram. The reduced row echelon form is a standard way to describe a linear subspace. The Ferrers diagram is a standard way to describe a partition of a given positive integer. Based on these two structures and the identifying vector of a subspace we will present a few representations for subspaces which will be the key for our enumerative encodings.

A $k$-dimensional subspace $X \in \mathbb{F}_{q}^{n}$ can be represented by a $k \times n$ generator matrix whose rows form a basis for $X$. To have a unique representation of a subspace, we use the following definition.

A $k \times n$ matrix with rank $k$ is in reduced row echelon form (RREF in short) if the following conditions are satisfied.

- The leading coefficient of a row is always to the right of the leading coefficient of the previous row.

- All leading coefficients are ones.

- Every leading coefficient is the only nonzero entry in its column.

We represent a subspace $X$ of a Grassmannian by its generator matrix in RREF. There is exactly one such matrix and it will be denoted by $R E(X)$.

Example 1: We consider the 3-dimensional subspace $X$ of $\mathbb{F}_{2}^{7}$ with the following eight elements.

$\begin{array}{llllllll}1) & (0 & 0 & 0 & 0 & 0 & 0 & 0) \\ 2) & (1 & 0 & 1 & 1 & 0 & 0 & 0) \\ 3) & (1 & 0 & 0 & 1 & 1 & 0 & 1) \\ 4) & (1 & 0 & 1 & 0 & 0 & 1 & 1) \\ 5) & (0 & 0 & 1 & 0 & 1 & 0 & 1) \\ 6) & (0 & 0 & 0 & 1 & 0 & 1 & 1) \\ 7) & (0 & 0 & 1 & 1 & 1 & 1 & 0) \\ 8) & (1 & 0 & 0 & 0 & 1 & 1 & 0)\end{array}$.

The generator matrix of $X$ in RREF is given by

$$
R E(X)=\left(\begin{array}{lllllll}
1 & 0 & 0 & 0 & 1 & 1 & 0 \\
0 & 0 & 1 & 0 & 1 & 0 & 1 \\
0 & 0 & 0 & 1 & 0 & 1 & 1
\end{array}\right) .
$$

Remark 2: It appears that designing an enumerative encoding of the Grassmannian based on this representation won't be efficient and we need to find other representations of a subspace for this purpose.

Each $k$-dimensional subspace $X$ of $\mathbb{F}_{q}^{n}$ has an identifying vector $v(X)$ [7]. $v(X)$ is a binary vector of length $n$ and weight $k$, where the ones in $v(X)$ are in the positions (columns) where $R E(X)$ has the leading coefficients (of the rows).

Remark 3: We can consider an identifying vector $v(X)$ for some $k$-dimensional subspace $X$ as a characteristic vector of a $k$-subset. This coincides with the definition of rankand order-preserving map $\phi$ from $\mathcal{G}_{q}(n, k)$ onto the lattice of subsets of an $n$-set, given by Knuth [9] and discussed by Milne [10].

Example 2: Consider the 3-dimensional subspace $X$ of Example 1 Its identifying vector is $v(X)=1011000$.

Remark 4: For a representation of a $k$-dimensional subspace $X$ we only need $v(X)$ and the $k \times(n-k)$ matrix formed by the columns of $R E(X)$ which correspond to the zeroes in $v(X)$.

Remark 5: A somewhat less compact way to represent a $k$-dimensional subspace $X$ is to form a $(k+1) \times n$ matrix where the first row is the identifying vector, $v(X)$, and the last $k$ rows form the RREF of $X, R E(X)$. We will see in the sequel that this representation will be very useful in our encoding algorithms.

Example 3: Consider the subspace $X$ of Example 11 Its representation by a $(k+1) \times n$ matrix is given by

$$
\left(\begin{array}{lllllll}
1 & 0 & 1 & 1 & 0 & 0 & 0 \\
1 & 0 & 0 & 0 & 1 & 1 & 0 \\
0 & 0 & 1 & 0 & 1 & 0 & 1 \\
0 & 0 & 0 & 1 & 0 & 1 & 1
\end{array}\right)
$$

A partition of a positive integer $m$ is a representation of $m$ as a sum of positive integers. The partition function $p(m)$ is the number of partitions of $m$ [15], [16].

Example 4: One of the possible partitions of 21 is $6+5+$ $5+3+2$ and $p(21)=792$.

A Ferrers diagram $\mathcal{F}$ represents a partition as a pattern of dots with the $i$-th row having the same number of dots as the $i$-th term in the partition [15], [16]. A Ferrers diagram satisfies the following conditions.

- The number of dots in a row is at most the number of dots in the previous row.

- All the dots are shifted to the right of the diagram.

Let $|\mathcal{F}|$ denote the size of $\mathcal{F}$, i.e., the number of dots in $\mathcal{F}$.

Example 5: For the partition of Example 4 the Ferrers diagram $\mathcal{F},|\mathcal{F}|=21$, is given by

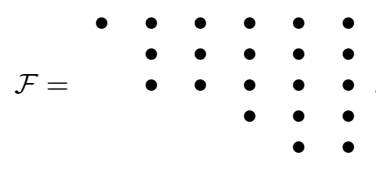

The echelon Ferrers form of a vector $v$ of length $n$ and weight $k, E F(v)$, is the $k \times n$ matrix in RREF with leading entries (of rows) in the columns indexed by the nonzero entries of $v$ and " $"$ in all entries which do not have terminal zeroes or ones. A " " will be called in the sequel a dot. The dots of this matrix form the Ferrers diagram of $E F(v)$. If we substitute elements of $\mathbb{F}_{q}$ in the dots of $E F(v)$ we obtain a $k$-dimensional subspace $X$ of $\mathcal{G}_{q}(n, k) . E F(v)$ will be called also the echelon Ferrers form of $X$.

Example 6: The echelon Ferrers form of the vector $v=$ 1011000 is

$$
E F(v)=\left(\begin{array}{lllllll}
1 & \bullet & 0 & 0 & \bullet & \bullet & \bullet \\
0 & 0 & 1 & 0 & \bullet & \bullet & \bullet \\
0 & 0 & 0 & 1 & \bullet & \bullet & \bullet
\end{array}\right)
$$

The Ferrers tableaux form of a subspace $X$, denoted by $\mathcal{F}(X)$, is obtained by assigning the values of $R E(X)$ in the Ferrers diagram of $E F(v(X))$.

Remark 6: $\mathcal{F}(X)$ defines a representation of $X$. 
Example 7: For the subspace $X$, given in Example 1 whose echelon Ferrers form given in 6 the Ferrers tableaux form is

$$
\mathcal{F}(X)=\begin{array}{llll}
0 & 1 & 1 & 0 \\
& 1 & 0 & 1 \\
& 0 & 1 & 1
\end{array} .
$$

\section{ENCODING BASED ON FERRERS TABLEAUX FORMS}

In this section we present an encoding of the Grassmannian based on the Ferrers tableaux form representation of $k$ dimensional subspaces. The number of dots in a Ferrers diagram of a $k$-dimensional subspace is at most $k \cdot(n-k)$. It can be embedded in a $k \times(n-k)$ box. We define a lexicographic order of such Ferrers diagrams, which induces an order of subspaces in the Grassmannian, and then apply the enumerative encoding to all $k$-dimensional subspaces.

The order that we define in the sequel is based on the following theorem [15] which shows the connection between the number of $k$-dimensional subspaces of $\mathbb{F}_{q}^{n}$, denoted by the $q$-ary Gaussian coefficient $\left[\begin{array}{l}n \\ k\end{array}\right]_{q}$, and partitions.

Theorem 2: For any given integers $k$ and $n, k \leq n$,

$$
\left[\begin{array}{l}
n \\
k
\end{array}\right]_{q}=\sum_{\ell=0}^{k(n-k)} \alpha_{\ell} q^{\ell},
$$

where the coefficient $\alpha_{\ell}$ is the number of partitions of $\ell$ whose Ferrers diagrams fit in a box of size $k \times(n-k)$.

\section{A. Encoding of Ferrers Diagrams}

Let $\mathcal{F}$ be a Ferrers diagram of size $m$ embedded in a $k \times(n-k)$ box. We represent $\mathcal{F}$ by an integer vector of length $n-k,\left(\mathcal{F}_{n-k}, \ldots, \mathcal{F}_{2}, \mathcal{F}_{1}\right)$, where $\mathcal{F}_{i}$ is equal to the number of dots in the $i$-th column of $\mathcal{F}, 1 \leq i \leq n-k$, where we number the columns from right to left. Note that $\mathcal{F}_{i+1} \leq \mathcal{F}_{i}, 1 \leq i \leq n-k-1$.

Let $\mathcal{F}$ and $\widetilde{\mathcal{F}}$ be two Ferrers diagrams of the same size. We say that $\mathcal{F}<\widetilde{\mathcal{F}}$ if $\mathcal{F}_{i}>\widetilde{\mathcal{F}}_{i}$ for the least index $i$ such that $\mathcal{F}_{i} \neq \widetilde{\mathcal{F}}_{i}$, i.e., in the least column where they have a different number of dots, $\mathcal{F}$ has more dots than $\widetilde{\mathcal{F}}$.

Let $N_{m}\left(\mathcal{F}_{j}, \ldots, \mathcal{F}_{2}, \mathcal{F}_{1}\right)$ be the number of Ferrers diagrams of size $m$ embedded in a $k \times(n-k)$ box, for which the first $j$ columns are given by $\left(\mathcal{F}_{j}, \ldots, \mathcal{F}_{2}, \mathcal{F}_{1}\right)$. The number of dots in column $j$ of $\mathcal{F}$ is at most $\mathcal{F}_{j-1}$. Hence, by (1) the lexicographic index $i n d_{m}$ of $\mathcal{F}$ among all the Ferrers diagrams with the same size $m$ is given by

$$
\operatorname{ind}_{m}(\mathcal{F})=\sum_{j=1}^{n-k} \sum_{a=\mathcal{F}_{j}+1}^{\mathcal{F}_{j-1}} N_{m}\left(a, \mathcal{F}_{j-1}, \ldots, \mathcal{F}_{2}, \mathcal{F}_{1}\right) \text {, }
$$

where we define $\mathcal{F}_{0}=k$.

Note that $0 \leq i n d_{m}(\mathcal{F}) \leq \alpha_{m}-1$, where $\alpha_{m}$ is defined in Theorem 2

Let $p(m, k, \eta)$ be the number of Ferrers diagrams of size $m$ which are embedded in a $k \times \eta$ box, i.e., $p(m, k, n-k)=\alpha_{m}$. The following lemma can be easily verified.

Lemma 1: $p(m, k, \eta)$ satisfies the following recurrence relation:

$$
p(m, k, \eta)=p(m-k, k, \eta-1)+p(m, k-1, \eta)
$$

$$
p(m, k, \eta)=p(k \eta-m, k, \eta),
$$

with the initial conditions

$$
\begin{gathered}
p(m, k, \eta)=0, \text { if } m<0 ; \\
p(m, 1, \eta)=1, \text { if } m \leq \eta ; \\
p(m, k, 1)=1, \text { if } m \leq k .
\end{gathered}
$$

Remark 7: Since $p(m, k, \eta)=p(k \eta-m, k, \eta)$, we can assume that $m \leq \frac{k \eta}{2}$.

Now, using the definition of $p(m, k, \eta)$ we can calculate the size of $N_{m}\left(\mathcal{F}_{j}, \ldots, \mathcal{F}_{2}, \mathcal{F}_{1}\right)$.

Lemma 2:

$$
N_{m}\left(\mathcal{F}_{j}, \ldots, \mathcal{F}_{2}, \mathcal{F}_{1}\right)=p\left(m-\sum_{i=1}^{j} \mathcal{F}_{i}, \mathcal{F}_{j}, n-k-j\right) .
$$

Lemma 2 implies that if we can calculate $p(m, k, \eta)$ efficiently then we can calculate efficiently $i n d_{m}(\mathcal{F})$ for Ferrers diagram of size $m$ embedded in a $k \times(n-k)$ box.

Given an index $i$, in a similar way to the inverse algorithm of Cover we can design an inverse algorithm to find the Ferrers diagram $\mathcal{F}$ such that $i=i n d_{m}(\mathcal{F})$.

Now, we can define an order of all Ferrers diagrams embedded in a $k \times(n-k)$ box.

For two Ferrers diagrams $\mathcal{F}$ and $\widetilde{\mathcal{F}}$, we say that $\mathcal{F}<\widetilde{\mathcal{F}}$ if one of the following conditions holds

- $|\mathcal{F}|>|\widetilde{\mathcal{F}}|$

- $|\mathcal{F}|=|\widetilde{\mathcal{F}}|$, and $i n d_{|\mathcal{F}|}(\mathcal{F})<\operatorname{ind}_{|\widetilde{\mathcal{F}}|}(\widetilde{\mathcal{F}})$.

Example 8: For the three Ferrers diagrams $\mathcal{F}, \widetilde{\mathcal{F}}$, and $\widehat{\mathcal{F}}$

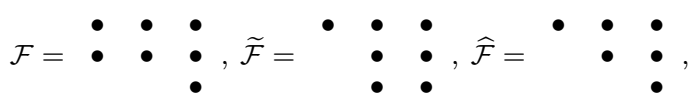

we have $\widetilde{\mathcal{F}}<\mathcal{F}<\widehat{\mathcal{F}}$.

\section{B. Order based on the Ferrers Tableaux Forms}

Let $X, Y \in \mathcal{G}_{q}(n, k)$ be two $k$-dimensional subspaces, $R E(X)$ and $R E(Y)$ the related RREFs. Let $v(X)$ and $v(Y)$ be the identifying vectors of $X$ and $Y$, respectively, and $\mathcal{F}_{X}, \mathcal{F}_{Y}$ the related Ferrers diagrams of $E F(v(X))$ and $E F(v(Y))$. Let $x_{1}, x_{2}, \ldots, x_{\left|\mathcal{F}_{X}\right|}$ and $y_{1}, y_{2}, \ldots, y_{\left|\mathcal{F}_{Y}\right|}$ be the entries of Ferrers tableaux forms $\mathcal{F}(X)$ and $\mathcal{F}(Y)$, respectively. The entries of a Ferrers tableaux form are numbered from right to left, and from top to bottom.

We say that $X<Y$ if one of the following conditions holds

- $\mathcal{F}_{X}<\mathcal{F}_{Y}$;

- $\mathcal{F}_{X}=\mathcal{F}_{Y}$, and $\left(x_{1}, x_{2}, \ldots, x_{\left|\mathcal{F}_{X}\right|}\right)<\left(y_{1}, y_{2}, \ldots, y_{\left|\mathcal{F}_{Y}\right|}\right)$.

Example 9: Let $X, Y, Z, W \in \mathcal{G}_{2}(6,3)$ which are given by

$$
\begin{aligned}
& R E(X)=\left(\begin{array}{llllll}
1 & 0 & \mathbf{1} & \mathbf{1} & 0 & \mathbf{1} \\
0 & 1 & \mathbf{1} & \mathbf{1} & 0 & \mathbf{1} \\
0 & 0 & 0 & 0 & 1 & \mathbf{1}
\end{array}\right), \quad \mathcal{F}(X)=\begin{array}{lll}
1 & 1 & 1 \\
1 & 1 & 1 \\
&
\end{array} \\
& R E(Y)=\left(\begin{array}{llllll}
1 & \mathbf{1} & 0 & 0 & \mathbf{0} & \mathbf{1} \\
0 & 0 & 1 & 0 & \mathbf{0} & \mathbf{0} \\
0 & 0 & 0 & 1 & \mathbf{1} & \mathbf{1}
\end{array}\right), \quad \mathcal{F}(Y)=\begin{array}{lll}
1 & 0 & 1 \\
0 & 0 \\
1 & 1
\end{array}
\end{aligned}
$$




$$
\begin{aligned}
& R E(Z)=\left(\begin{array}{llllll}
1 & \mathbf{1} & 0 & \mathbf{1} & 0 & \mathbf{1} \\
0 & 0 & 1 & \mathbf{1} & 0 & \mathbf{1} \\
0 & 0 & 0 & 0 & 1 & \mathbf{0}
\end{array}\right), \quad \mathcal{F}(Z)=\begin{array}{lll}
1 & 1 & 1 \\
& 1 & 1 \\
& & 0
\end{array} \\
& R E(W)=\left(\begin{array}{llllll}
1 & \mathbf{1} & 0 & \mathbf{1} & 0 & \mathbf{1} \\
0 & 0 & 1 & \mathbf{1} & 0 & \mathbf{1} \\
0 & 0 & 0 & 0 & 1 & \mathbf{1}
\end{array}\right), \quad \mathcal{F}(W)=\begin{array}{lll}
1 & 1 & 1 \\
& 1 & 1
\end{array} .
\end{aligned}
$$

From Example 8 we have $\mathcal{F}_{Y}<\mathcal{F}_{X}<\mathcal{F}_{Z}=\mathcal{F}_{W}$. Since $\left(z_{1}, z_{2}, \ldots, z_{\left|\mathcal{F}_{Z}\right|}\right)=(1,1,0,1,1,1)<\left(w_{1}, w_{2}, \ldots, w_{\left|\mathcal{F}_{W}\right|}\right)=$ $(1,1,1,1,1,1)$ it follows that $Y<X<Z<W$.

\section{Encoding Based on the Ferrers Tableaux Forms}

Now, we use the order defined above and Theorem 2 for enumerative encoding of $\mathcal{G}_{q}(n, k)$. Let $\{x\}$ be the integer value of vector $x=\left(x_{1}, \ldots, x_{\left|\mathcal{F}_{X}\right|}\right)$ and let $\{i\}_{q}$ be the base $q$ representation of the integer $i$.

Theorem 3: Let $X \in \mathcal{G}_{q}(n, k), \mathcal{F}_{X}$ be the Ferrers diagram of $\operatorname{EF}(v(X)), x_{1}, x_{2}, \ldots, x_{\left|\mathcal{F}_{X}\right|}$ be the entries of $\mathcal{F}(X)$. Then the index $\operatorname{Ind}_{1}(X)$, by the order based on the Ferrers tableaux forms, is given by

$$
\operatorname{Ind}_{1}(X)=\sum_{i=\left|\mathcal{F}_{X}\right|+1}^{k(n-k)} \alpha_{i} q^{i}+i n d_{\left|\mathcal{F}_{X}\right|}\left(\mathcal{F}_{X}\right) q^{\left|\mathcal{F}_{X}\right|}+\{x\},
$$

where $\alpha_{i}$ is defined in Theorem 2 and $i n d_{\left|\mathcal{F}_{X}\right|}$ is given by (2).

Now, an index $i$ is given. The following algorithm returns a subspace $X \in \mathcal{G}_{q}(n, k)$ such that $\operatorname{Ind}_{1}(X)=i$.

\section{Inverse algorithm:}

Step 1: If $i<q^{k(n-k)}$ then $\left|\mathcal{F}_{X}\right|=k(n-k)$; assign the values of $\{i\}_{q}$ to $\mathcal{F}(X)$ and stop; otherwise set $i=$ $i-q^{k(n-k)}$.

Step 2: For $1 \leq j \leq k(n-k)$, if $i<\alpha_{k(n-k)-j} q^{k(n-k)-j}$, then $\left|\mathcal{F}_{X}\right|=k(n-k)-j, \mathcal{F}_{X}=i n d_{\left|\mathcal{F}_{X}\right|}^{-1}\left(\left\lfloor\frac{i}{q^{k(n-k)-j}}\right\rfloor\right)$; assign the values of $\left\{i-\left\lfloor\frac{i}{q^{k(n-k)-j}}\right\rfloor q^{k(n-k)-j}\right\}_{q}$ to $\mathcal{F}(X)$ and stop; otherwise set $i=i-\alpha_{k(n-k)-j} q^{k(n-k)-j}$.

Theorem 4: The complexity of the encoding/decoding based on the Ferrers tableaux forms is $O\left(k^{5 / 2}(n-k)^{5 / 2}\right)$.

\section{RREF AND IDENTIFYING VECTOR ENCODING}

In this section we provide another method for enumerative encoding of the Grassmannian, based on the representation of a subspace $X \in \mathcal{G}_{q}(n, k)$ by a $(k+1) \times n$ matrix whose first row is $v(X)$ and the other $k$ rows form $R E(X)$. First, we define the lexicographic order in the Grassmannian based on this representation and then we apply enumerative encoding to the Grassmannian based on this representation.

\section{A. Order based on the Extended Representation}

Let $X \in \mathcal{G}_{q}(n, k)$ be a $k$-dimensional subspace. The extended representation $E X T(X)$ of $X$ is a $(k+1) \times$ $n$ matrix obtained by combining the identifying vector $v(X)=\left(v(X)_{n}, \ldots, v(X)_{1}\right)$ and the RREF $R E(X)=$ $\left(X_{n}, \ldots, X_{1}\right)$, as follows

$$
\operatorname{EXT}(X)=\left(\begin{array}{cccc}
v(X)_{n} & \ldots & v(X)_{2} & v(X)_{1} \\
X_{n} & \ldots & X_{2} & X_{1}
\end{array}\right)
$$

Note, that $v(X)_{i}$ is the most significant bit of the column vector $\left(\begin{array}{c}v(X)_{i} \\ X_{i}\end{array}\right)$.

Let $X, Y \in \mathcal{G}_{q}(n, k)$ and $\operatorname{EXT}(X), \operatorname{EXT}(Y)$ be the extended representations of $X$ and $Y$, respectively. Let $i$ be the least index such that $\operatorname{EXT}(X)$ and $\operatorname{EXT}(Y)$ have different columns. We say that $X<Y$ if $\left\{\begin{array}{c}v(X)_{i} \\ X_{i}\end{array}\right\}<$ $\left\{\begin{array}{c}v(Y)_{i} \\ Y_{i}\end{array}\right\}$.

Example 10: For $X, Y \in \mathcal{G}_{2}(6,3)$ whose $\operatorname{EXT}(X)$, and $\operatorname{EXT}(Y)$ are given by

$$
\begin{aligned}
& \operatorname{EXT}(X)=\left(\begin{array}{llllll}
1 & 1 & 1 & 0 & 0 & 0 \\
1 & 0 & 0 & 0 & 1 & 0 \\
0 & 1 & 0 & 0 & 0 & 0 \\
0 & 0 & 1 & 1 & 0 & 0
\end{array}\right) \\
& \operatorname{EXT}(Y)=\left(\begin{array}{llllll}
1 & 1 & 0 & 0 & 1 & 0 \\
1 & 0 & 0 & 0 & 0 & 0 \\
0 & 1 & 0 & 0 & 0 & 0 \\
0 & 0 & 0 & 0 & 1 & 0
\end{array}\right),
\end{aligned}
$$

we have $X<Y$.

\section{B. Enumerative Encoding Based on Extended Representation}

Let $N\left(\begin{array}{ccc}v_{j} & \ldots & v_{1} \\ X_{j} & \ldots & X_{1}\end{array}\right)$ be the number of elements in $\mathcal{G}_{q}(n, k)$ for which the first $j$ columns in the extended representation are given by $\left(\begin{array}{ccc}v_{j} & \ldots & v_{1} \\ X_{j} & \ldots & X_{1}\end{array}\right)$.

Remark 8: We view all the $q$-ary vectors of length $k+1$ as our finite alphabet. Let $S$ be the set of all $q$-ary $(k+$ 1) $\times n$ matrices which form extended representations of some $k$-dimensional subspaces. Now, we can use Cover's method to encode the Grassmannian. In this setting note that $N\left(\begin{array}{ccc}v_{j} & \ldots & v_{1} \\ X_{j} & \ldots & X_{1}\end{array}\right)$ is equivalent to $n_{S}\left(x_{1}, x_{2}, \ldots, x_{j}\right)$, where $x_{i}=\left(\begin{array}{c}v_{i} \\ X_{i}\end{array}\right)$.

Lemma 3:

$$
N\left(\begin{array}{ccc}
v_{j} & \ldots & v_{1} \\
X_{j} & \ldots & X_{1}
\end{array}\right)=\left[\begin{array}{c}
n-j \\
k-\sum_{i=1}^{j} v_{i}
\end{array}\right]_{q} .
$$

Theorem 5: Let $X \in \mathcal{G}_{q}(n, k)$ be represented by

$$
\operatorname{EXT}(X)=\left(\begin{array}{cccc}
v_{n} & \cdots & v_{2} & v_{1} \\
X_{n} & \cdots & X_{2} & X_{1}
\end{array}\right)
$$

Then the lexicographic index of $X$ is given by

$\operatorname{Ind}_{2}(X)=\sum_{j=1}^{n}\left(v_{j} q^{k-w_{j-1}}+\left(1-v_{j}\right) \frac{\left\{X_{j}\right\}}{q^{w_{j-1}}}\right)\left[\begin{array}{c}n-j \\ k-w_{j-1}\end{array}\right]_{q}$

where $w_{j-1}$ denotes the weight of the first rightmost $j-1$ entries of $v(X)$, i.e., $w_{j-1}=\sum_{\ell=1}^{j-1} v_{\ell}$.

Example 11: Let $X \in \mathcal{G}_{2}(6,3)$ be given by

$$
\operatorname{EXT}(X)=\left(\begin{array}{llllll}
0 & 1 & 0 & 1 & 1 & 0 \\
0 & 1 & 1 & 0 & 0 & 1 \\
0 & 0 & 0 & 1 & 0 & 0 \\
0 & 0 & 0 & 0 & 1 & 1
\end{array}\right)
$$

By Theorem 5 we have

$$
\begin{gathered}
\operatorname{Ind}_{2}(X)=5 \cdot\left[\begin{array}{l}
5 \\
3
\end{array}\right]_{2}+2^{3} \cdot\left[\begin{array}{l}
4 \\
3
\end{array}\right]_{2}+2^{2} \cdot\left[\begin{array}{l}
3 \\
2
\end{array}\right]_{2} \\
+1 \cdot\left[\begin{array}{l}
2 \\
1
\end{array}\right]_{2}+2 \cdot\left[\begin{array}{l}
1 \\
1
\end{array}\right]_{2}=928 .
\end{gathered}
$$


Now suppose that an index $i$ is given. The following algorithm finds a subspace $X \in \mathcal{G}_{q}(n, k)$ such that $\operatorname{Ind}_{2}(X)=i$.

Inverse algorithm: Set $i_{0}=i$.

For $j=1,2, \ldots, n$ do:

- if $w_{j-1} \geq k$ then set $v(X)_{j}=0, X_{j}=\{0\}_{q}$, and $i_{j}=i_{j-1}$;

- otherwise

- if $i_{j-1} \geq q^{k-w_{j-1}}\left[\begin{array}{c}n-j \\ k-w_{j-1}\end{array}\right]_{q}$ then set $v(X)_{j}=$ $1, \quad X_{j}=\left\{q^{w_{j-1}}\right\}_{q}$, and $i_{j}=i_{j-1}-$ $q^{k-w_{j-1}}\left[\begin{array}{c}n-j \\ k-w_{j-1}\end{array}\right]_{q} ;$

- otherwise let val $=\left\lfloor i_{j-1} /\left[\begin{array}{c}n-j \\ k-w_{j-1}\end{array}\right]_{q}\right\rfloor$ and set $v(X)_{j}=0, X_{j}=\left\{\mathrm{val} \cdot q^{w_{j-1}}\right\}_{q}$, and $i_{j}=i_{j-1}-$ val $\cdot\left[\begin{array}{c}n-j \\ k-w_{j-1}\end{array}\right]_{q}$.

Theorem 6: The complexity of the encoding/decoding based on the extended representation is $O(n k(n-$ $k) \log n \log \log n)$.

\section{Combination of the Encoding Methods}

The only disadvantage of the Ferrers tableaux form encoding is the computation of the $\alpha_{i}$ 's and $\operatorname{ind}_{\left|\mathcal{F}_{X}\right|}\left(\mathcal{F}_{X}\right)$ in Theorem 3. This is the reason for its relatively higher complexity. The advantage of this encoding is that once these values are known, the algorithm becomes trivial. Our solutions for the computation of the $\alpha_{i}$ 's and $i n d_{\left|\mathcal{F}_{X}\right|}\left(\mathcal{F}_{X}\right)$ are relatively not efficient and this is the main reason why we turned to enumerative encoding based of the RREF and the identifying vector of a subspace. The only disadvantage of this enumerative encoding is the computation of the Gaussian coefficients in Theorem 5. It appears that a combination of the two methods is more efficient from the efficiency of each one separately. The complexity will remain $O(n k(n-k) \log n \log \log n)$, but the constant will be considerably reduced in the average. This can be done if there won't be any need for the computation of the $\alpha_{i}$ 's and the computation of $i n d_{\left|\mathcal{F}_{X}\right|}\left(\mathcal{F}_{X}\right)$ will be simple.

We note that most of the $k$-dimensional subspaces have a Ferrers diagram with a large number of dots. We will encode these subspaces by the Ferrers tableaux form encoding and the other subspaces by the extended representation encoding. We will decide on a set $S_{\mathcal{F}}$ of Ferrers diagrams which will be used for the Ferrers tableaux form encoding. They will be taken by a decreasing number of dots among all the Ferrers diagrams which can be embedded in a $k \times(n-k)$ box.

We define a new function $\widehat{I n d}$ in the following way:

$$
\widehat{\operatorname{Ind}}(X)=\left\{\begin{array}{cc}
\operatorname{Ind}_{1}(X) & \mathcal{F}_{X} \in S_{\mathcal{F}} \\
\operatorname{Ind}_{2}(X)+\Delta_{X} & \text { otherwise }
\end{array},\right.
$$

where $\Delta_{X}$ is the number of subspaces formed from $S_{\mathcal{F}}$, which are lexicographically succeeding $X$ by the extended representation ordering. Similarly we will define an inverse algorithm.

\section{Conclusion and Future Research}

Three methods for enumerative encoding of the Grassmannian are presented. The first is based on the Ferrers tableaux form of subspaces. The second is based on the representation of subspaces by their identifying vector and reduced row echelon form. The complexity of the second method is superior on the complexity of the first one. The third method which is a combination of the first two reduces in average the constant in the first term of the complexity for the second method. Improving on these methods is a problem for future research.

Enumerative encoding of the Grassmannian is based on representation and order of subspaces. Each such order defines a lexicographic code [17] with prescribed minimum distance (for two subspaces $X, Y \in \mathcal{G}_{q}(n, k)$ the distance between $X$ and $Y$ is defined by $d(X, Y)=\operatorname{dim} X+\operatorname{dim} Y-$ $2 \operatorname{dim}(X \cap Y)$ [1]). It appears that some of these lexicodes are the best known. For example, based of the Ferrers tableaux form ordering we found a code with minimum distance 4 and size 4605 in $\mathcal{G}_{2}(8,4)$ which is the largest known. Considering lexicographic codes in the Grassmannian is a topic for future research. There are some computational aspects involve in this computation and this is currently under consideration.

\section{ACKNOWLEDGMENT}

This work was supported in part by the Israel Science Foundation (ISF), Jerusalem, Israel, under Grant No. 230/08.

\section{REFERENCES}

[1] R. Koetter and F. R. Kschischang, "Coding for errors and erasures in random network coding," IEEE Trans. Inform. Theory, vol. 54, no. 8, pp. 3579-3591, August 2008.

[2] S. T. Xia and F. W. Fu, "Johnson type bounds on constant dimension codes," arxiv.org/abs/0709.1074.

[3] T. Etzion and A. Vardy, "Error-correcting codes in projective space", proc. Int. Symp. on Inform. Theory, Toronto, pp. 871-875, July 2008.

[4] F. Manganiello, E. Gorla, and J. Rosenthal, "Spread codes and spread decoding in network coding", proc. of Int. Symp. on Inform. Theory, pp. 881-885, July 2008 .

[5] D. Silva, F. R. Kschischang, and R. Koetter, "A Rank-metric approach to error control in random network coding," IEEE Trans. Inform. Theory, vol. IT-54, pp. 3951-3967, September 2008.

[6] M. Gadouleau and Z. Yan, "Constant-rank codes and their connection to constant-dimension codes," arxiv.org/abs/0803.2262.

[7] T. Etzion and N. Silberstein, "Error-correcting codes in projective space via rank-metric codes and Ferrers diagrams", arxiv.org/abs/0807.4846.

[8] V. Skachek, "Recursive code construction for random network," arxiv.org/abs/0806.3650.

[9] D. E. Knuth, "Subspaces, subsets. and partitions ," J. Combin. Theory, vol. 10, pp. 178-180, 1971.

[10] S. Milne, "Mappings of subspaces into subsets ," J. Combin. Theory, vol. Series A, vol. 33, pp. 36-47, 1982.

[11] R. Ahlswede, H. K. Aydinian, and L. H. Khachatrian, "On perfect codes and related concepts," Designs, Codes, Crypt., vol. 22, 221-237, 2001.

[12] M. Schwartz, T. Etzion, "Codes and anticodes in the Grassman graph," Journal of Combinatorial Theory, Series A, vol. 97, pp. 27-42, 2002.

[13] A. Kohnert and S. Kurz, "Construction of large constant dimension codes with a prescribed minimum distance," arxiv.org/abs/0807.3212.

[14] T.M. Cover, "Enumerative source encoding," IEEE Trans. Inform. Theory, vol. IT-19, no. 1, pp. 73-77, Jan. 1973.

[15] J. H. van Lint and R. M. Wilson, A course in Combinatorics, Cambridge University Press, 1992.

[16] G. E. Andrews and K. Eriksson, Integer Partitions, Cambridge University Press, 2004.

[17] J. H. Conway and N. J. A. Sloane, "Lexicographic codes: errorcorrecting codes from game theory," IEEE Trans. Inform. Theory, vol. IT-32, pp. 337-348, May 1986. 